\title{
Removal of Cells from Body Fluids by Magnetic Separation in Batch and Continuous Mode: Influence of Bead Size, Concentration, and Contact Time
}

\author{
Nils Bohmer, ${ }^{\dagger, \|}$ Nino Demarmels, ${ }^{\dagger, \|}$ Elena Tsolaki, ${ }^{\ddagger}$ Lukas Gerken, ${ }^{\dagger}$ Kerda Keevend, ${ }^{\dagger}$ Sergio Bertazzo, ${ }^{\ddagger \oplus}$ \\ Marco Lattuada, ${ }^{\S(0)}$ and Inge K. Herrmann* ${ }^{*}{ }^{\dagger}$

\begin{abstract}
${ }^{\dagger}$ Materials Meet Life Department, Swiss Federal Laboratories for Materials Science and Technology (Empa), Lerchenfeldstrasse 5, CH-9014, St. Gallen, Switzerland

${ }^{*}$ Department of Medical Physics and Biomedical Engineering, University College London (UCL), Malet Place Engineering Building, London, WC1E 6BT, United Kingdom

${ }^{\S}$ Department of Chemistry, University of Fribourg, Chemin du Musée 9, CH-1700, Fribourg, Switzerland
\end{abstract}

\section{Supporting Information}

\begin{abstract}
The magnetic separation of pathogenic compounds from body fluids is an appealing therapeutic concept. Recently, removal of a diverse array of pathogens has been demonstrated using extracorporeal dialysis-type devices. The contact time between the fluid and the magnetic beads in such devices is limited to a few minutes. This poses challenges, particularly if large compounds such as bacteria or cells need to be removed. Here, we report on the feasibility to remove cells from body fluids in a continuous dialysis type of setting. We assessed tumor cell removal efficiencies from physiological fluids with or without white blood cells using a range of different magnetic bead sizes $(50-4000 \mathrm{~nm})$, concentrations, and contact times. We show that tumor cells can be quantitatively removed from body fluids within acceptable times (1-2 min) and bead concentrations $(0.2 \mathrm{mg}$ per $\mathrm{mL}$ ). We further present a mathematical model to describe the minimal bead number concentration needed to remove a certain number of cells, in the presence of competing nonspecific uptake. The present study paves the way for investigational studies to assess the therapeutic potential of cell removal by magnetic blood purification in a dialysis-like setting.
\end{abstract}

KEYWORDS: blood cells, blood purification, magnetic particles, mathematical modeling, nanoparticles, tumor cells

\section{INTRODUCTION}

Magnetic separation offers a direct way for the removal of disease-causing factors from body fluids. Magnetic beads functionalized by a capturing moiety are employed to selectively bind to target compounds and are then subsequently removed by magnetic separation. ${ }^{1-3}$ A variety of beads, mostly based on iron oxide, iron, and iron carbide, have successfully been applied to capture target compounds. Removal of a wide variety of different substances, including metal ions, ${ }^{4,5}$ small molecule drugs, ${ }^{6,7}$ and proteins ${ }^{6}$ has been demonstrated in various in vitro and in vivo models. Recently, the concept has been extended to remove bacteria from body fluids in diagnostic, ${ }^{8}$ therapeutic, ${ }^{9}$ and theranostic ${ }^{10}$ settings. A similar concept based on micron-sized magnetic beads has been employed for magnetic cell isolation in cell culture and diagnostics for decades already. ${ }^{11-13}$ Although micrometer-sized beads work well for diagnostic applications, $\mathrm{Xu}$ and colleagues have highlighted significant drawbacks of microparticles, such as low surface area (and binding capacity) and slow binding. ${ }^{14}$ In the same report, they also have demonstrated promising separation efficiencies of tumor cells using $30-\mathrm{nm}$ iron oxide beads in a batch type setting. The removal of tumor cells from body fluid holds some promise ${ }^{15}$ and various studies have been conducted on the isolation of circulating tumor cells (CTCs). ${ }^{1-3,16-20}$ For example, McDonald and colleagues have demonstrated in an early study that ovarian cancer progression in mice is 10 -fold lower when migratory tumor cells are removed by magnetic filtration from intraperitoneal fluids. ${ }^{21}$ Most of the reported results in the literature aim at the diagnosis ${ }^{1,16,17,20}$ and monitoring of cancer via isolation of CTCs from the blood of patients by microfluidic techniques. ${ }^{1,3,16-18,20}$ However, rapid therapeutic removal of cells by magnetic separation in a continuous process has not yet been investigated systematically, and intrinsic process limitations remain unclear.

In a continuous extracorporeal process, similar to a dialysis setting, the contact time between the beads and the fluid containing the target cells is limited to a few minutes. ${ }^{22}$ For efficient binding, long contact times would be beneficial, but the 
receptor binding process is in competition with the uptake of particles into phagocytic cells. Additionally, long contact times compromise the overall process throughput. The effective removal of tumor cells relies on high particle-tumor cells collision frequencies and specific binding of magnetic clusters to tumor cells in the presence of proteins and other cells, such as white blood cells. Here, we investigated the technical feasibility and limitations for the therapeutic removal of cells from body fluids using a magnetic blood purification process in batch and continuous mode. We assessed the influence of bead size and concentration, and bead-body fluid contact times on tumor cell separation efficiency in the presence of white blood cells, employing a combination of experimental and theoretical methods.

\section{MATERIALS AND METHODS}

Magnetic Beads and Antibodies. Protein A/G coated Bio-Adembeads from Ademtech (04631, Lot 15G022-1 and Lot 15L012-1) were used. For size- and time-dependence experiments, beads from Creative Diagnostic Absolute Mag were used in addition to the Adembeads. For all bead functionalizations, the EpCAM antibody from Abcam (ab187372 (VU-1D9]) was used. EpCAM-FITC (ab112067 (VU-1D9)) was used for analysis of the EpCAM epitope on Caco-2 cells. Transmission electron micrographs of beads were collected using a JEOL 100 Plus TEM. Hydrodynamic sizes of the particles were measured using a dynamic light scattering (DLS) instrument (ZetaSizer, $90^{\circ}$ configuration).

Bead Functionalization. Bio-Adembeads were functionalized according to the manufacturer's protocol for IgG cross-linking. The incubation time was increased to $1 \mathrm{~h}$. Bead pelleting was done in a 500- $\mu \mathrm{L}$ Eppendorf tube with a cubic Neodymium magnet, $4.6 \times$ $3 \times 1 \mathrm{~cm}$, acquired from supermagnete.ch. Creative Diagnostics beads with a bead concentration of $10 \mathrm{mg}$ per $\mathrm{mL}$ were functionalized by adding, e.g., $1 \mu \mathrm{g}$ of antibody to $10 \mu \mathrm{L}$ of beads. The solution was then incubated for $1 \mathrm{~h}$ at $1000 \mathrm{rpm}$ on the Heidolph Titramax 101 shaker. Subsequently, the tube was placed on the magnet until a bead pellet formed. The supernatant was discarded and the pellet was resuspended in PBS. The washing was repeated twice.

Cell Lines. For all separation experiments the Caco-2 cell line (ACC169), acquired from Deutsche Sammlung von Mikroorganismen and Zellkulturen $\mathrm{GmbH}$ was used. Undifferentiated Caco-2 cells were cultured in cell culture medium (Minimum Essential Medium Eagle (MEM), Sigma-Aldrich, M2279) containing 10\% fetal calf serum (FCS, Sigma-Aldrich, F9665), 1\% non-essential amino acids (Sigma-Aldrich, M7145), 1\% penicillin-streptomycin-neomycin (Sigma-Aldrich, P4083), and $1 \%$ L-glutamine (Sigma-Aldrich, G7513). Cells were maintained at $37{ }^{\circ} \mathrm{C}$ and $5 \% \mathrm{CO}_{2}$ in humidified atmosphere and routinely subcultured twice a week at $70-80 \%$ confluence by treatment with $0.5 \%$ trypsinEDTA (Sigma-Aldrich, T3924). THP-1 cells (TIB-202) were acquired from American Type Culture Collection. THP-1 monocytic cells were cultured in suspension in RPMI-1640 medium (Sigma-Aldrich, R0883) supplemented with $10 \%$ FCS, $1 \%$ penicillin-streptomycin-neomycin, and $1 \%$ L-glutamine and routinely subcultured once a week.

AnnexinV/PI Assay. To exclude acute cytotoxicity of the nanoparticles, apoptosis and necrosis induced were assessed. For detection of apoptotic and necrotic cells the FITC Annexin V Apoptosis Detection Kit I (556547, BD Biosciences) was used. Caco-2 cells were seeded in 24-well plates (50000 cells/well or $2680 \mathrm{cells} / \mathrm{cm}^{2}$ ) and grown to $50-60 \%$ confluency before adding stimuli or nanoparticles. Cells were incubated in duplicates for $24 \mathrm{~h}$ with EpCAMfunctionalized magnetic beads in a concentration of $0.4 \mathrm{mg}$ per $\mathrm{mL}$ $\left(0.11 \mathrm{mg} / \mathrm{cm}^{2}\right)$ and $0.04 \mathrm{mg}$ per $\mathrm{mL}\left(0.01 \mathrm{mg} / \mathrm{cm}^{2}\right)$. For short incubation, mimicking the therapeutic time scale, magnetic beads were spiked directly into the cell suspension during the staining procedure. Silica nanoparticles (Aerosil200, Evonik) in the same concentrations served as a positive control for nanoparticle toxicity. Cadmium sulfate $(100 \mu \mathrm{M})$ was added for $3 \mathrm{~h}$ to untreated cells as a control for necrosis while staurosporine $(10 \mu \mathrm{M})$ was added for $4 \mathrm{~h}$ as a control for the induction of apoptosis. After incubation with stimuli, cells were harvested by treatment with Trypsin/EDTA and stained according to the manufacturer's instructions. Cells were assessed with the Gallios flow cytometer (10 000 events gated in forward/side scatter or $2 \mathrm{~min}$ max. acquisition time) and the fluorescence signals of PI and AnnexinV-FITC were detected in FL4 or FL1, respectively.

Blood. Blood was donated in-house by healthy volunteers $(n=5)$. Every donor signed a written consent approved by the ethical commission of St. Gallen (EKSG 12/111). After having obtained written informed consent, $2-5 \mathrm{~mL}$ of citrated blood was withdrawn using a 20G needle. All donations were collected in a Vacutainer buffered with sodium citrate at $0.109 \mathrm{M}$ (Belliver Industrial Estate, Plymouth, UK, Lot 40340010). Only volunteers who had not taken any drugs known to affect platelet function for 2 weeks prior to donation were included.

Cell Staining. Specific cell staining was performed to allow discrimination between the different cell types after separation experiments in flow cytometry. The THP-1 cells were stained using Hoechst 33342, dissolved in PBS at a concentration of $0.5 \mu \mathrm{g}$ per $\mathrm{mL}$, and incubated for $20 \mathrm{~min}$ at $4{ }^{\circ} \mathrm{C}$. Caco-2 cells were stained with CellTracker Green CMFDA (Thermo Fisher Scientific, C2925), dissolved in prewarmed cell culture medium at a concentration of $60 \mu \mathrm{M}$, and incubated for $15 \mathrm{~min}$ at $37^{\circ} \mathrm{C}$. After incubation the cells were centrifuged at $200 \mathrm{~g}$ for $5 \mathrm{~min}$ and resuspended in prewarmed MEM cell culture medium.

Cell Separation Experiments. Separation experiments were done in prewarmed MEM with $10 \%$ fetal calf serum, $1 \%$ non-essential amino acids, $1 \%$ penicillin-streptomycin-neomycin, and $1 \%$ L-glutamine. Beads were functionalized according to the abovedescribed procedure. Cells were resuspended in culture medium at a concentration of 100000 cells per mL. For experiments with human leukocytes, blood was collected and part of the blood was incubated with VersaLyse buffer to lyse the red blood cells. Leukocytes were counted and used immediately or, when fixed leukocytes were required, the pellet was fixed in 4\% PFA. The leukocytes were resuspended in cell culture medium, which contained 100000 stained Caco- 2 cells per mL. Then, $25 \mu \mathrm{L}$ of cell culture medium or $25 \mu \mathrm{L}$ of beads was added to the samples and incubated at $37{ }^{\circ} \mathrm{C}$ on a linear shaker. Magnetic separation was achieved by holding a cubic neodymium magnet next to the 1.5 -mL Eppendorf for $1 \mathrm{~min}$. The entire supernatant was then aspirated. The pellet was resuspended in cell culture medium. This allowed analysis of the total number of cells, on the one hand the separated residual cells and on the other hand the unseparated supernatant. Counting beads (CountBrightAbsolute Counting Beads from Molecular Probes) were then added and samples were analyzed in a flow cytometer.

Flow Cytometric Analysis. A Beckmann \& Coulter Gallios TM Flow Cytometer was used in combination with Kaluza Analysis Software. Samples that contained CellTracker Green stained cells were excited at $488 \mathrm{~nm}$ and measured with a 525/540 nm bandpass. Samples containing Hoechst 33342 stained cells were excited at $405 \mathrm{~nm}$ and measured with a 540/550 nm bandpass. Counting beads were excited at $635 \mathrm{~nm}$ and measured at $755 \mathrm{~nm}$. All samples were measured in Micronic 1.4-mL tubes.

To ensure comparable cell counts in every sample, data acquisition was stopped after 5000 counting beads gated in fluorescence channel 7 (FL7). Cells were gated according to their specific fluorescent label in complex mixtures or depending on their forward and side scatter signal when only one cell type was involved.

TEM Sample Preparation and Imaging. For electron microscopy, cells (white blood cells (WBC) and tumor cells (TC), incubated with particles for $1 \mathrm{~min}$ and $5 \mathrm{~h}$ ) were gently washed with prewarmed PBS and fixed with $4 \%$ methanol-free paraformaldehyde (PFA) overnight in the fridge. Pellets were then washed with $\mathrm{ddH}_{2} \mathrm{O}$ $(3 \mathrm{x})$ and cacodylate buffer $(0.1 \mathrm{M})(2 \mathrm{x})$ and stained with $2 \%$ osmium tetroxide and $1.5 \%$ potassium ferricyanide for $1 \mathrm{~h}$. Pellets were washed with $\mathrm{ddH}_{2} \mathrm{O}$ and then gradually dehydrated using an ethanol gradient $(40 \%, 50 \%, 60 \%, 70 \%, 80 \%, 90 \%, 95 \%, 100 \%(3 x))$. Cell pellets were then embedded in epoxy resin (EPON 812), according to procedures described in the manufacturer's protocol. Resin blocks were cured in the oven for $72 \mathrm{~h}$, trimmed with a razor blade and then sectioned in $100-\mathrm{nm}$ sections using an ultramicrotome. The thin sections were imaged in a JEOL 100 Plus TEM at $80 \mathrm{keV}$. 
Continuous Experiment. A blood purification device was assembled at a scale of 1:10 of a previously constructed magnetic blood purification device. ${ }^{22}$ Two pumps (Ismatech Pump ISM 833C Type 335193) were used. Flow rates were set to $1.5 \mathrm{~mL}$ per $\mathrm{min}$ for the main tube and beads were added at a 1:10 dilution. Functionalized beads were pumped in a PharMedBPT orange/yellow tube into the main silicon TR60 2/4MM tube to reach a final bead concentration of $0.4 \mathrm{mg}$ per $\mathrm{mL}$. The silicon tube was replaced for each new sample. For experiments including a mixer, a pearl chain mixer (Fluidic 658 from Microfluidic chipshop) was connected to the tubing. The volume of the entire device was $5 \mathrm{~mL}$. The volume between mixer and magnets was $3 \mathrm{~mL}$, resulting in a contact time of $2 \mathrm{~min}$. The beads were separated from the fluid flow by using two neodymium magnets, assembled using an in-house 3D-printed scaffold. Final cell concentrations were analyzed in flow cytometry by mixing $300 \mu \mathrm{L}$ of the cell suspension after separation with $25 \mu \mathrm{L}$ of the counting beads. Acquisition was stopped after 5000 counting beads and the residual cell number was determined for every sample.

Mathematical Model. To quantify the time required for magnetic particles to bind to cells, and consequently gain information about the time evolution of the fraction of cells with a given number magnetic particles bound to their surface, a mathematical model based on population balance equations has been used. This model, which is similar to the one used in our previous work, ${ }^{10}$ relies on the solution of mass balances for the overall concentration of free magnetic particles, as well as for the concentration of cells carrying an arbitrary number $i$ of magnetic particles bound to them. Let us call $N$ the number concentration of unbound magnetic particles, and $C_{i}$ the concentration of cells with $i$ particles adsorbed. The mass balance for magnetic particles reads

$$
\frac{\mathrm{d} N}{\mathrm{~d} t}=-N \cdot \sum_{i=0}^{M-1} K_{i} \cdot C_{i}
$$

where $M$ is the maximum number of particles that can be attached on a cell. Equation 1 states that the adsorption of particles onto cells is described as a bimolecular event, proportional to the concentration of free particles and to the concentration of cells onto which particles are going to attach. The "rate constant $K_{i}$ ", corresponding to the rate of particle attaching on a cell with having already $i$ particles bound to its surface, is given by the following expression, valid for diffusion-limited events:

$$
K_{i}=\frac{2 k_{b} T}{3 \eta}\left(R_{C}+R_{M P}\right)\left(\frac{1}{R_{C}}+\frac{1}{R_{M P}}\right)\left(1-\frac{i}{M}\right)
$$

In Equation 2, $R_{C}$ is the radius of the cell, $R_{M P}$ is the radius of the magnetic particles, $T$ is the absolute temperature, $k_{B}$ is the Boltzmann constant, and $\eta$ is the viscosity of the medium in which particles and cells move. The last term in the equation is the correction accounting for the fact that a cell with $i$ particles bound to it has only a portion of its surface available for further particle attachment. When $M$ particles are attached, no more particles can be attached, since the model does not foresee the possibility of multiple layers of particles attached to the surface of a cell. The mass balance equations for cells are

$$
\begin{aligned}
& \frac{\mathrm{d} C_{0}}{\mathrm{~d} t}=-K_{0} \cdot N \cdot C_{0} \\
& \frac{\mathrm{d} C_{i}}{\mathrm{~d} t}=K_{i-1} \cdot N \cdot C_{i-1}-K_{i} \cdot N \cdot C_{i} \\
& \frac{\mathrm{d} C_{M}}{\mathrm{~d} t}=-K_{M-1} \cdot N \cdot C_{M-1}
\end{aligned}
$$

We can write two conservation equations. The first is the conservation of cells

$$
\sum_{i=0}^{M} C_{i}=C_{T}
$$

where $C_{T}$ is the total number of cells, which is equal to the initial number of cells with zero particles adsorbed on them.
The second is the conservation of particles

$$
\sum_{i=1}^{M} i \cdot C_{i}+N=N_{0}
$$

where $N_{0}$ is the initial and total number concentration of magnetic particles.

It is possible to find a solution in closed form of Equations 1- 5, as shown in the Supporting Information. The closed form solution makes the calculations of all relevant quantities (such as the time evolution of the average number of particles bound to a cell) very easy.

The model can be further extended to the case of unspecific binding. In this case, it is assumed that there are two populations of cells: those to which magnetic particles are supposed to bind, and the second population, to which particles bind unspecifically, and with a considerably lower rate. Equation 3 still holds for the cells to which magnetic particles are supposed to bind. A similar set of equations can be written in the case of cells with unspecific binding. Overall, the balance equations for the two cell populations are

$$
\begin{aligned}
& \frac{\mathrm{d} C_{1,0}}{\mathrm{~d} t}=-K_{1,0} \cdot N \cdot C_{1,0} \\
& \frac{\mathrm{d} C_{1, i}}{\mathrm{~d} t}=K_{1, i-1} \cdot N \cdot C_{1, i-1}-K_{1, i} \cdot N \cdot C_{1, i} \\
& \frac{\mathrm{d} C_{1, M}}{\mathrm{~d} t}=-K_{1, M-1} \cdot N \cdot C_{1, M-1} \\
& \frac{\mathrm{d} C_{2,0}}{\mathrm{~d} t}=-K_{2,0} \cdot N \cdot C_{2,0} \\
& \frac{\mathrm{d} C_{2, i}}{\mathrm{~d} t}=K_{2, i-1} \cdot N \cdot C_{2, i-1}-K_{2, i} \cdot N \cdot C_{2, i} \\
& \frac{\mathrm{d} C_{2, M}}{\mathrm{~d} t}=-K_{2, M-1} \cdot N \cdot C_{2, M-1}
\end{aligned}
$$

where the symbols with subscript 1 and 2 refer to cells belonging to population 1 (specific) and 2 (unspecific), respectively. The corresponding balance for the particles becomes

$$
\frac{\mathrm{d} N}{\mathrm{~d} t}=-N \cdot\left(\sum_{i=0}^{M_{1}-1} K_{1, i} \cdot C_{1, i}+\sum_{i=0}^{M_{2}-1} K_{2, i} \cdot C_{2, i}\right)
$$

And the rate constants are given by

$$
\begin{aligned}
K_{1, i} & =\frac{2 k_{b} T}{3 \eta}\left(R_{1, C}+R_{M P}\right)\left(\frac{1}{R_{1, C}}+\frac{1}{R_{M P}}\right)\left(1-\frac{i}{M_{1}}\right) \\
K_{2, i} & =\frac{2 k_{b} T}{3 \eta W}\left(R_{2, C}+R_{M P}\right)\left(\frac{1}{R_{2, C}}+\frac{1}{R_{M P}}\right)\left(1-\frac{i}{M_{2}}\right)
\end{aligned}
$$

where $M_{1}$ and $M_{2}$ are the maximum number of particles that can bind to the two populations of cells. If the sizes of the two cells are identical, then $M_{1}=M_{2}$. The only difference between the two rate constants is the presence of the $W$ factor in the denominator of the second eq 8 . This factor, assumed to be $W \gg 1$, corrects for unspecific binding, which is assumed to be much slower than the specific binding. $1 / W$ represents the probability that a collision between a magnetic particle and a cell leads to an unspecific binding event. The total cells and particles conservation equations read

$$
\begin{aligned}
& \sum_{i=0}^{M_{1}} C_{1, i}=C_{1, T} \\
& \sum_{i=0}^{M_{2}} C_{2, i}=C_{2, T} \\
& \sum_{i=1}^{M_{1}} i \cdot C_{1, i}+\sum_{i=1}^{M_{2}} i \cdot C_{2, i}+N=N_{0}
\end{aligned}
$$


The solution of equations $6-9$ has been done partially analytically, partially numerically, as described in the Supporting Information.

\section{RESULTS AND DISCUSSION}

To investigate the receptor-mediated specific binding of magnetic particles to cells and the efficiency of the magnetic separation process, EpCAM-positive tumor cells were chosen as a)

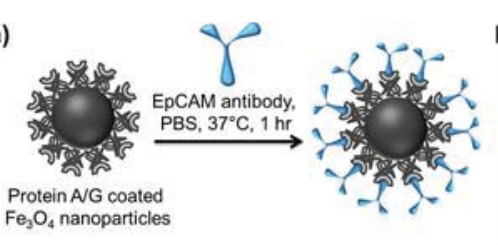

MNP-PAG

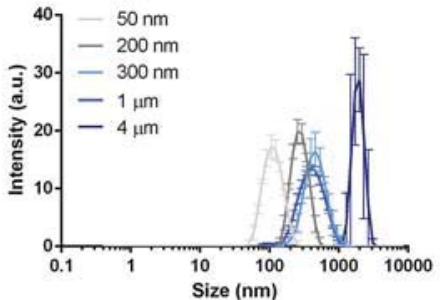

b)

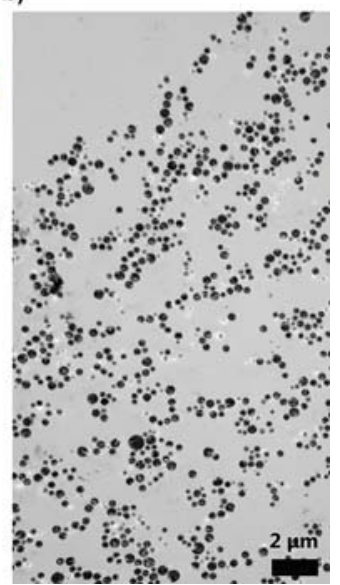

Figure 1. (a) Protein A-coated magnetic nanoparticles (or Protein A/G hybrid-coated 300-nm nanoparticles) where functionalized with EpCAM antibodies. (b) Transmission electron micrographs of 300-nm protein A/G hybrid-coated nanoparticles. (c) Dynamic light scattering measurements of nanoparticle clusters of different size. a model cell line. EpCAM is a surface marker that is widely expressed by different tumor cells and has been used to separate tumor cells in diagnostic settings for many years already. ${ }^{17,23}$ EpCAM antibody functionalized beads were assembled using Protein A (or Protein A/G hybrid)-coated magnetic clusters with cluster diameters of $50 \mathrm{~nm}, 200 \mathrm{~nm}, 300 \mathrm{~nm}, 1 \mu \mathrm{m}$, and $4 \mu \mathrm{m}$ (according to the manufacturer; Figure 1a). Transmission electron micrographs of the $300-\mathrm{nm}$ clusters show a uniform size and shape of the particles (Figure 1b). Dynamic light scattering (DLS) measurements show relatively narrow size distributions and average hydrodynamic sizes of $106( \pm 0.7) \mathrm{nm}$, $264( \pm 5) \mathrm{nm}, 367( \pm 1) \mathrm{nm}, 432( \pm 5) \mathrm{nm}$, and 3209( \pm 460$) \mathrm{nm}$ in water (Figure 1b). Hydrodynamic sizes in cell culture medium were comparable to the ones in water (see SI Figure S1). These DLS sizes differ considerably from the expected size provided by the supplier for 1 and $4 \mu \mathrm{m}$ particles. However, this may be explained by anisotropic particle shapes and inaccuracies in the estimate of the refractive index.

An EpCAM-positive and an EpCAM-negative cell line were selected for initial bead-cell interaction studies. The EpCAM positivity of Caco- 2 cells and the low EpCAM expression on THP-1 cells were confirmed by flow cytometry (Figure 2a,b). Then, 300-nm beads with and without EpCAM-antibody attached were added to a suspension of Caco- 2 cells (100000 cells per $\mathrm{mL}$ ) at a concentration of $0.4 \mathrm{mg}$ per $\mathrm{mL}$. The compatibility of the beads for at least the duration of a typical experiment and up to $24 \mathrm{~h}$ was demonstrated in a membrane integrity assay (Figure 2c). No significant effect on cell viability was observed and viability remained $\geq 90 \%$ for at least $24 \mathrm{~h}$ of a)

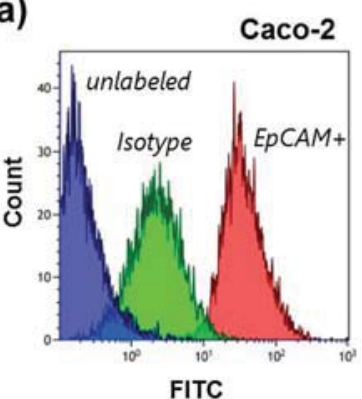

c)

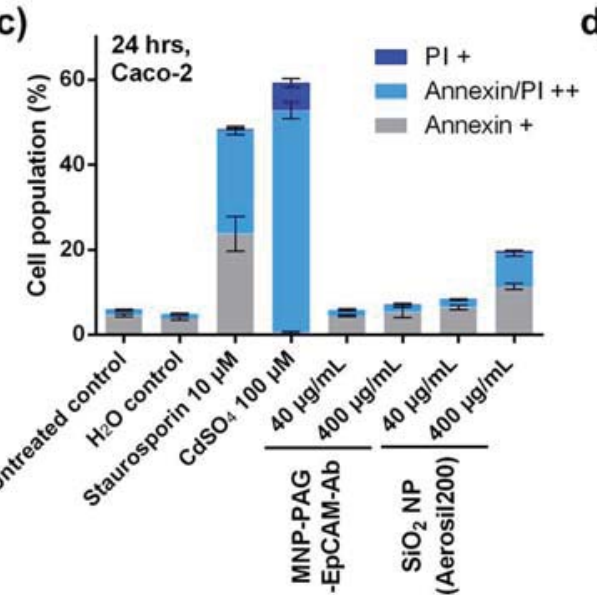

b)
THP-1

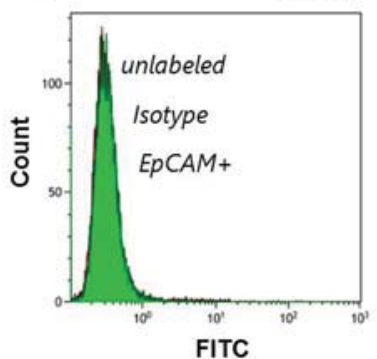

d)

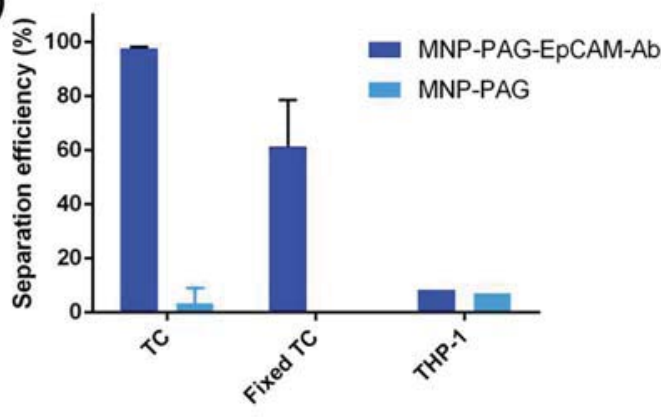

Figure 2. Cell characterization and interactions between magnetic beads and cells. EpCAM expression on (a) EpCAM-positive Caco-2 cells and (b) EpCAM-negative THP-1 cells. (c) Flow cytometry analysis of PI and Annexin stained Caco-2 cells following exposure to magnetic beads $(300 \mathrm{~nm})$ for $24 \mathrm{~h}$ and comparison to silica nanoparticles (Aerosil200) $(n=2)$. (d) Tumor cell separation efficiency using magnetic beads functionalized with EpCAM and beads without antibodies and an incubation time of $10 \min (n=2)$. 
incubation time. The nonspecific interaction of protein $A / G$ coated beads (without EpCAM-antibody) with Caco-2 cells was investigated (Figure 2d). Less than $5 \%$ of tumor cells were separated when beads without EpCAM-antibody were used. For EpCAM functionalized beads, separation efficiencies of $>95 \%$ were reached using bead concentrations of $0.4 \mathrm{mg}$ per $\mathrm{mL}$ and contact times of $10 \mathrm{~min}$. These results show that there is very little nonspecific interaction between cells and beads without antibody for incubation times up to $10 \mathrm{~min}$. Additionally, an analogous experiment with (EpCAM-negative) THP-1 cells shows that $<10 \%$ of THP- 1 cells are removed by the EpCAM-antibody functionalized magnetic beads. To differentiate between the competitive receptor binding and endocytosis processes, separation efficiencies of fixed and unfixed tumor cells were compared. When PFA-fixed Caco-2 cells were used, separation efficiencies were slightly reduced and reached $60 \%$ for EpCAM-antibody functionalized beads. Less than $1 \%$ of PFA-fixed cells were removed when beads without antibody were employed.

Separation efficiencies of EpCAM-positive Caco-2 cells by EpCAM-antibody-functionalized beads were then assessed in the presence of EpCAM-negative cells. First, a human monocyte cell line (THP-1) with comparatively low phagocytic activity was used as a model system. Binding of EpCAM-coated magnetic beads to EpCAM-negative THP-1 cells was found to be $<10 \%$ and separation efficiencies of Caco- 2 cells in the presence of THP-1 cells were $>95 \%$.
To assess the influence of magnetic bead size on tumor cell binding and uptake into phagocytic cells, different bead sizes ( $50 \mathrm{~nm}, 200 \mathrm{~nm}, 300 \mathrm{~nm}, 1 \mu \mathrm{m}$, and $4 \mu \mathrm{m}$ ) were compared at the same mass concentration in presence and absence of EpCAM-negative white blood cells (WBC, Figure 3a). Because undifferentiated THP-1 cells have a relatively low phagocytic activity, native white blood cells isolated from fresh human whole blood were employed to mimic conditions in human blood more realistically. At a concentration of $0.4 \mathrm{mg}$ per $\mathrm{mL}$, it becomes apparent that beads with sizes between $200 \mathrm{~nm}$ and $1 \mu \mathrm{m}$ are most efficient. This is in agreement with a study on bacteria removal from Kang et al. ${ }^{24}$ The $50-\mathrm{nm}$ beads are incompletely separated from the suspension by magnetic separation due to their weaker magnetic moment. Also, smaller beads are readily taken up by both WBC and tumor cells (TC), which possibly explains the slightly reduced separation efficacy in the presence of WBC for 50 and $200 \mathrm{~nm}$. On the other hand, the number of $4-\mu \mathrm{m}$ beads is too low to reach complete separation. This is in agreement with findings from $\mathrm{Xu}$ and colleagues, who report slow and inefficient binding of tumor cells by $3-5 \mu \mathrm{m}$ beads. $^{14}$

Next, the influence of bead concentration was investigated using 300-nm beads (Figure 3b). Experiments show that beads are in excess and that bead concentration of down to $0.2 \mathrm{mg}$ per $\mathrm{mL}$ leads to separation efficiencies of $>98 \%$. However, in the presence of white blood cells, with increasing incubation time, the minimum number of required beads to achieve a)

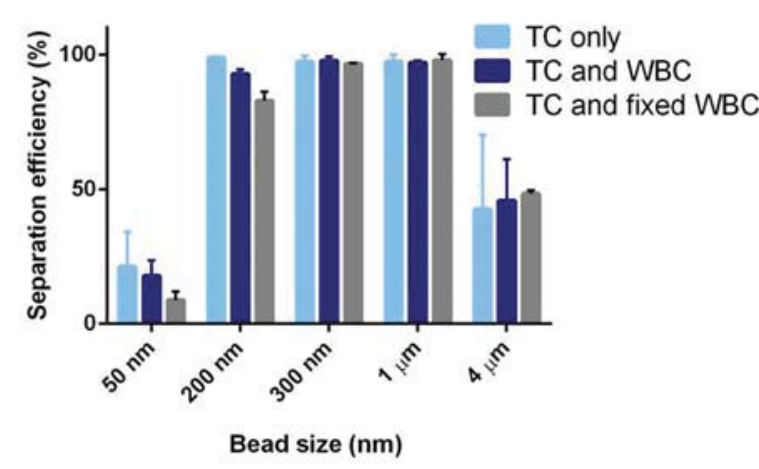

C)

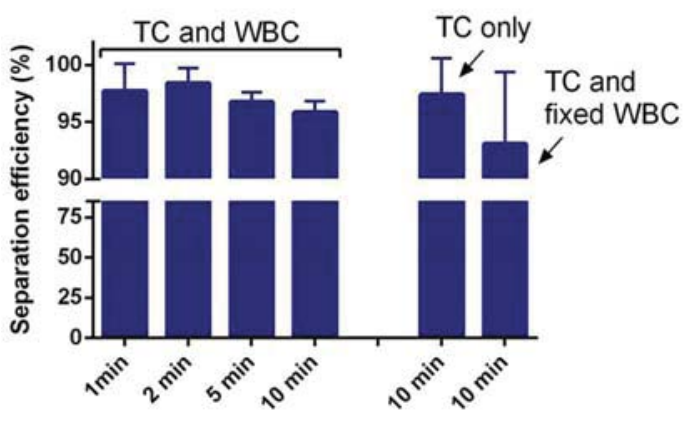

Contact time (min) b)

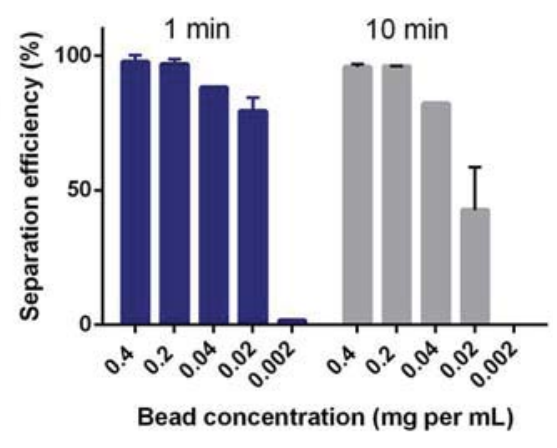

d)

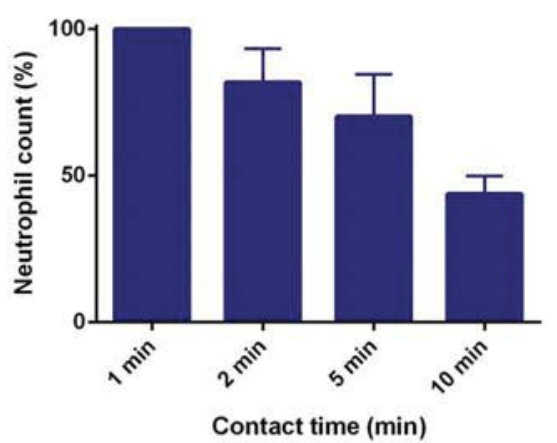

Figure 3. Study of the magnetic bead-cell interactions. Tumor cell separation efficiency as a function of (a) bead size in the presence of tumor cells only (TC), tumor cells and white blood cells (TC and WBC), and tumor cells and fixed white blood cells (TC and fixed white blood cells). (b) Separation efficiency using 300-nm magnetic beads as a function of bead concentration and incubation time ( 1 and 10 min). (c) The influence of bead-fluid contact time for particle concentrations of $0.2 \mathrm{mg}$ per $\mathrm{mL}$ for tumor cells in the presence of white blood cells. (d) The neutrophil count in the supernatant as a function of incubation time with 300 -nm magnetic beads at a concentration of $0.2 \mathrm{mg}$ per $\mathrm{mL}(n=3)$. 
a) $300 \mathrm{~nm}$ beads

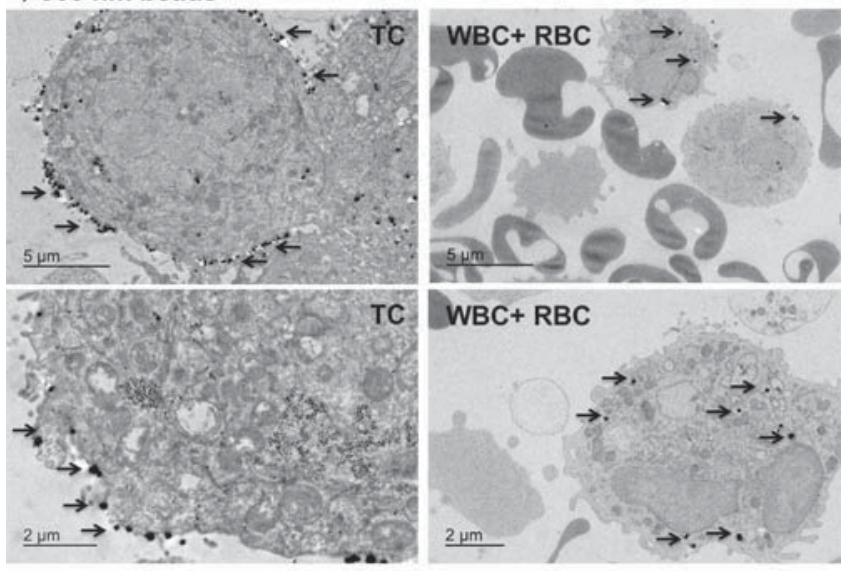

b) $4 \mu \mathrm{m}$ beads

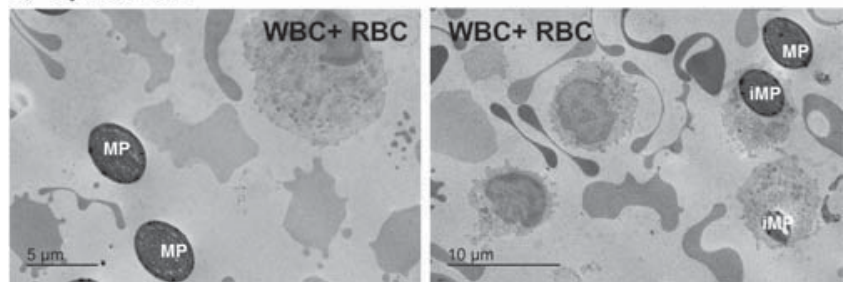

Figure 4. Particle uptake. Transmission electron micrographs of tumor cells (TC) and white blood cells (WBC) (and red blood cells (RBC)) incubated with $300-\mathrm{nm}$ and $4-\mu \mathrm{m}$ particles for $1 \mathrm{~min}$ or $5 \mathrm{~h}$, respectively. (a) The $300-\mathrm{nm}$ particles are localized primarily on the membrane of tumor cells after short incubation times. Some $300-\mathrm{nm}$ particles can be found inside both TC and WBC. Smaller particles are more readily internalized (black arrows). (b) Most of the $4-\mu \mathrm{m}$ particles are located extracellularly, however, occasionally, also $4-\mu \mathrm{m}$ particles (MP) can be found inside of cells (termed as intracellular microparticles (iMP)). quantitative separation is significantly higher, indicative of a competitive process of bead phagocytosis and binding of beads to tumor cells.

The influence of contact time between the beads and the cells has shown little effect of the contact time on TC separation efficiencies for bead concentration of $>0.2 \mathrm{mg}$ per $\mathrm{mL}$ (Figure 3c). However, a striking decrease in WBC, and particularly neutrophil counts, is observed with increasing contact times (Figure $3 \mathrm{~d}$ ). After $10 \mathrm{~min}$ of contact time, the neutrophil count drops by $50 \%$ of the initial count ( 1 million per $\mathrm{mL})$. Transmission electron micrographs confirm uptake of magnetic nanoparticles, with a preferential uptake of smaller particle (i.e., $300-\mathrm{nm}$ ) compared to $4-\mu \mathrm{m}$ particles (Figure $4 \mathrm{a}, \mathrm{b}$ ). Occasionally, also $4-\mu \mathrm{m}$ particles are found inside of the cells (Figure 4b). These findings indicate that blood-bead contact times should be limited to a few minutes in order to avoid uptake of magnetic particles into white blood cells.

Finally, we demonstrate efficacious removal of EpCAMpositive cells in a continuous process. We assembled a dialysis like system in analogy to the ones reported in previous studies. ${ }^{9,22,25}$ The volume of the device was $5 \mathrm{~mL}$ and fluid was pumped at a flow rate of $1.5 \mathrm{~mL}$ per min. Beads were added to the fluid stream at a final bead concentration of $0.4 \mathrm{mg}$ per $\mathrm{mL}$. In the continuous process, single pass tumor separation efficiency was found to be reduced (78.8\%) compared to the batch setting ( $>95 \%)$. To improve contact between beads and fluid, a microfluidic pearl chain mixer was introduced (Figure 5a,b). Using the mixer, separation efficiency was increased from $78.8 \%$ to $>92 \%$, even in the presence of physiological numbers of WBC (Figure 5c). The mixing of the beads with the cells is indeed a critical factor affecting separation performance.

Notably, we have used comparatively high number concentrations of tumor cells. The reason for that is mostly of technical nature, since such high concentrations allow reliable a)

Geometry A (w/o mixer)
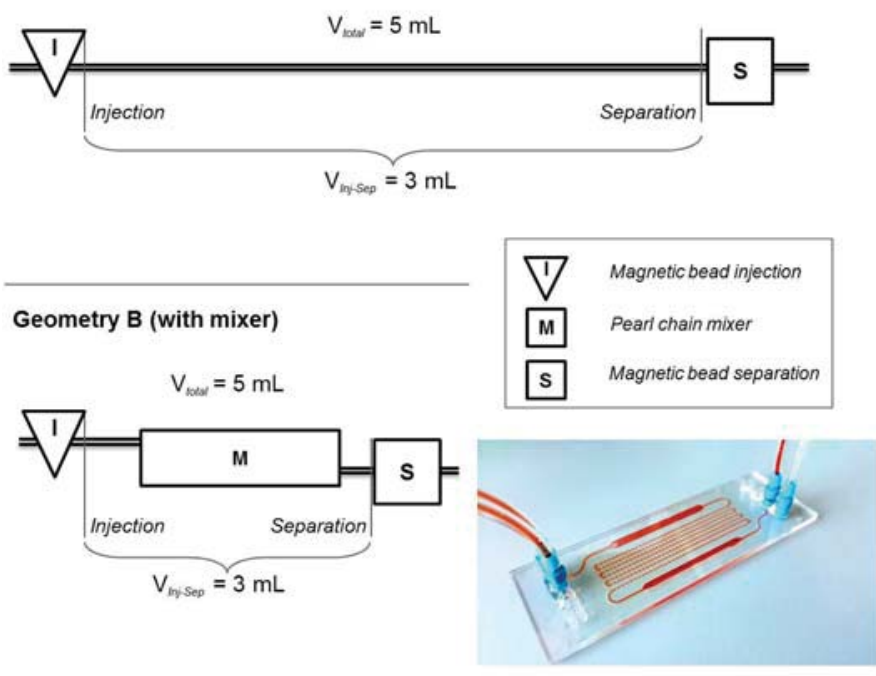

b)

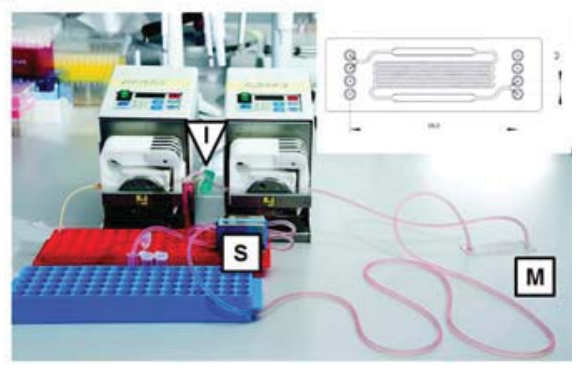

c)

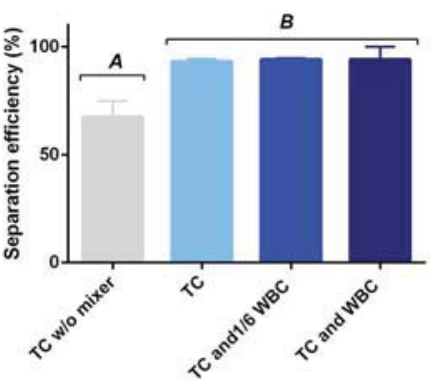

Figure 5. Study of the magnetic bead fluid contact. A device for continuous operation was assembled. (a) Two different geometries were investigated. Geometry A consisted of a silicon tube with a total volume of $5 \mathrm{~mL}$ and a volume of $3 \mathrm{~mL}$ between the bead injection point and the magnetic separator. Geometry B consisted of the same type of silicon tubing but the tubing was connected to a pearl chain microfluidic chip mixer. The volume between the injection point and the separator again was $3 \mathrm{~mL}(\mathrm{a}, \mathrm{b})$. (c) The tumor cell separation efficiencies compared for the two geometries $(n \geq 2)$. 

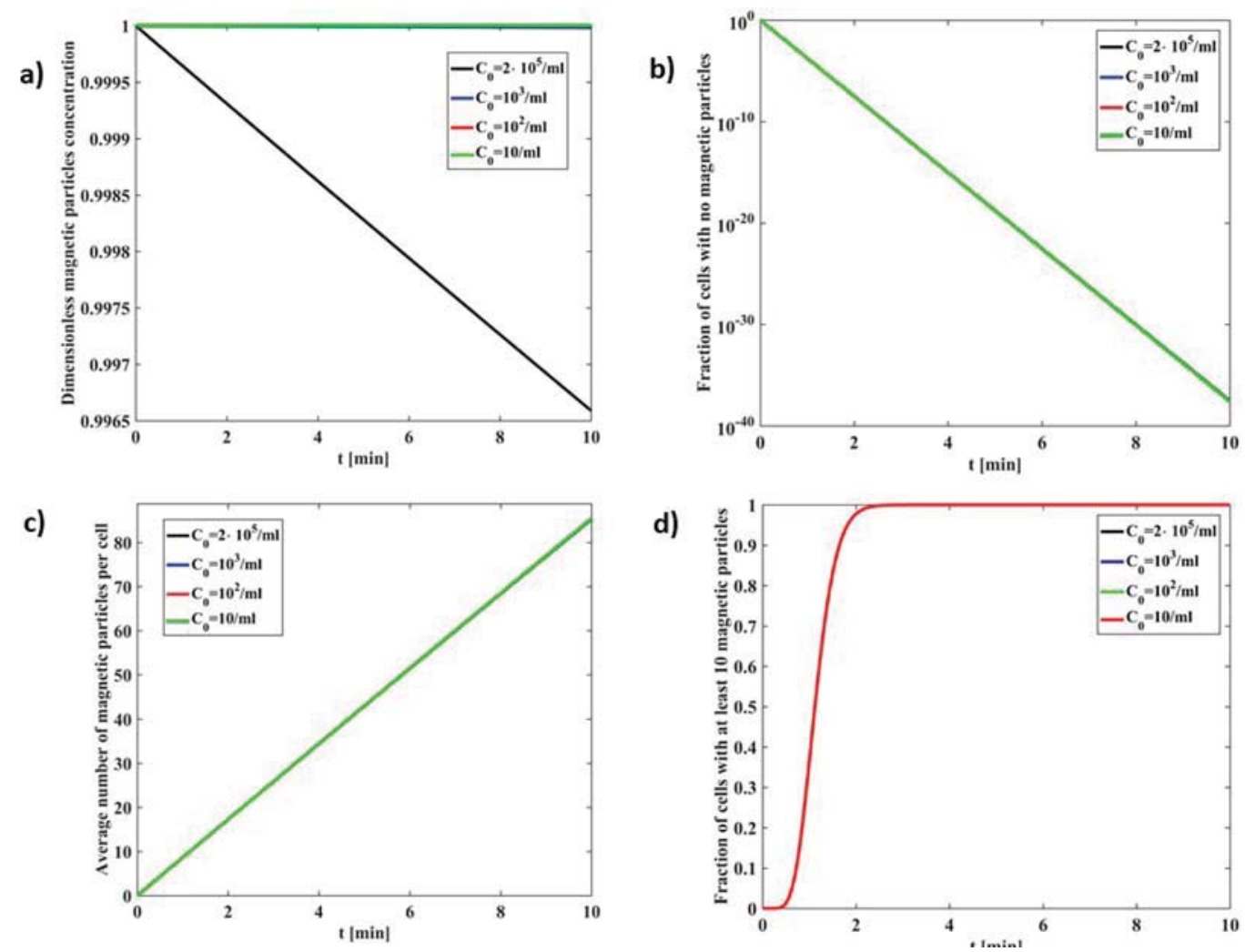

Figure 6. Mathematical modeling results for magnetic beads with a size of $300 \mathrm{~nm}$ and a number concentration of $5 \times 10^{9}$ beads per mL. (a) Change in magnetic nanoparticle concentration as a function of cell number $\left(10-10^{5}\right.$ per $\mathrm{mL}$ ) concentration and time. (b) The fraction of cells with no magnetic particles attached as a function of cell number concentration and time. (c) The average number of magnetic particles attached to a cell and (d) the time evolution of the fraction of cells carrying at least 10 particles.

cell counting by flow cytometry. The target cell concentration in certain clinical scenarios may be much lower, especially for circulating tumor cells where concentrations of $\leq 10$ cells per $\mathrm{mL}$ are reported, ${ }^{23,26}$ and this may affect separation performance. To address how target cell concentration will affect the removal efficiency, we calculated the estimated binding times based on a mathematical model. We used an estimated target cell size of $10 \mu \mathrm{m} \times 10 \mu \mathrm{m} \times 10 \mu \mathrm{m}$ and a bead concentration of $5 \times 10^{9}$ beads per $\mathrm{mL}$. The number of target cells was varied from 10 cells per $\mathrm{mL}$ to 100000 cells per mL. The modeling results show that beads are added in such great excess that the cell concentration only marginally affects the removal efficiency (Figure 6a,b). The number of beads per cell increases irrespective of the cell number in that case (Figure $6 c, d$ ). In particular, Figure $6 \mathrm{~d}$ shows the time evolution of cells with at least 10 magnetic beads. It is estimated that this is the requirement necessary to achieve good separation. ${ }^{24}$ One can see how in a couple of minutes all the cells contain at least 10 beads. Because particles are much smaller than cells in that case, the relative movements of particles are much faster, and cells can almost be considered as stationary phase. ${ }^{27}$ The rate-determining step in the binding process is the movement and the binding of nanoparticles to the cell surface, and the cell concentration thus has very little effect on the separation efficiency. However, nonspecific uptake may of course significantly affect the separation performance. We therefore adapted the model to take into account nonspecific uptake. To do so, we assumed that particles can also attach to nontarget cells, at a rate which is $\alpha$ times smaller than the rate of binding to target cells. We have performed a sensitivity analysis varying several parameters of the model in order to assess the effect of unspecific binding on the outcome of the separation. The results of such analyses are shown in SI Figures S2 and S3. The results are presented in terms of average number of cells bearing at least 10 particles as a function of the $\alpha$ value, for two extremely different ratios of concentrations of specific versus unspecific cells, $C_{1} / C_{2}$. Figure S2 shows four cases, corresponding to four different magnetic particles concentrations. One can observe that, for sufficiently low values of the parameter $\alpha$, the separation between specific and unspecific cells is almost complete. Not surprisingly, at $\alpha$ values sufficiently close to 1 , the separation becomes progressively poorer, until specificity is completely lost. As the particle concentration decreases, it appears that the number of cells with at least 10 particles that are present after $10 \mathrm{~min}$ decreases considerably. As a rule of thumb, in order to achieve an analogous performance, a decrease in particles concentration by about an order of magnitude can be compensated by an increase in contact time by 1 order of magnitude, as Figure S3 shows. In fact the fraction of cells with specific binding shown in Figures S2c and S3a is quite similar. In the former case, a concentration of particles of $5 \times 10^{7}$ per $\mathrm{mL}$ has been used for $10 \mathrm{~min}$, while in the latter case a particle concentration of $5 \times 10^{6}$ per $\mathrm{mL}$ for a time of $100 \mathrm{~min}$ has been used. Clearly the fact that the final results are not identical is due to the nonidentical consumption of particles, which becomes a problem when their concentration becomes sufficiently small, as Figure S2b shows, where a particle concentration of $5 \times 10^{6}$ per $\mathrm{mL}$ for a time of $1000 \mathrm{~min}$ has been used. The almost complete consumption of particles in the latter case can be seen in Figure S3d. Taken together, this model enables 
estimation of the minimal particle concentration required to remove a certain number of cells in a given time as a function of nonspecific interactions.

\section{CONCLUSIONS}

The present article demonstrates the conceptual feasibility of cell removal from body fluids in a continuous extracorporeal dialysis-type of setting. The contact between magnetic beads and target substances is critical, and can be enhanced by introducing a pearl chain mixer. Contact times higher than 2 min should be avoided in order to prevent nonspecific uptake into blood cells. Also, particle surface chemistry may be further optimized to improve specific cell binding and limit nonspecific interactions. The current setting could be easily scaled up to increase the throughput to $50-150 \mathrm{~mL}$ per min, similar to that of currently used blood purification devices. This study paves the way to investigate the therapeutic benefit of magnetic cell removal in conditions such as metastatic cancer or leukemia, where circulating tumor cells could be removed, or in autoimmune diseases.

\section{ASSOCIATED CONTENT}

S Supporting Information

The Supporting Information is available free of charge on the ACS Publications website at DOI: 10.1021/acsami.7b10140.

Figure S1. Hydrodynamic size of magnetic beads in water and cell culture medium. Further description of the mathematical model. Figures S2 and S3. Mathematical modeling results for magnetic beads with a size of $300 \mathrm{~nm}$ (PDF)

\section{AUTHOR INFORMATION}

\section{Corresponding Author}

*inge.herrmann@empa.ch.

\section{ORCID ๑}

Sergio Bertazzo: 0000-0003-4889-8190

Marco Lattuada: 0000-0001-7058-9509

Inge K. Herrmann: 0000-0002-3018-6796

\section{Author Contributions}

"N.B. and N.D. contributed equally as first authors.

\section{Author Contributions}

N.D. and N.B. performed the experimental work on cell removal, N.B. supervised the flow cytometry work, performed cytotoxicity assays, and helped write the manuscript, E.T. analyzed samples in TEM, L.G. performed dynamic light scattering measurements, K.K. helped with nanoparticle and TEM sample preparation, S.B. supervised TEM studies, M.L. performed the mathematical modeling, I.K.H. supervised the study and wrote the manuscript. All authors contributed to discussions and edited the manuscript.

\section{Notes}

The authors declare no competing financial interest.

\section{ACKNOWLEDGMENTS}

We acknowledge support from the Novartis Foundation for Medical-Biological Research. M.L. acknowledges financial support from the Swiss National Science Foundation (grant PP00P2_159258). N.B. acknowledges funding from the NanoScreen Materials Challenge cofunded by the Competence Centre for Materials Science and Technology (CCMX). We thank Ursina Tobler for assistance with blood collection from healthy volunteers.

\section{REFERENCES}

(1) Issadore, D.; Chung, J.; Shao, H.; Liong, M.; Ghazani, A. A.; Castro, C. M.; Weissleder, R.; Lee, H. Ultrasensitive Clinical Enumeration of rare Cells ex vivo using a $\mu$-Hall Detector. Sci. Transl. Med. 2012, 4 (141), 141ra92-141ra92.

(2) Mohamadi, R. M.; Besant, J. D.; Mepham, A.; Green, B.; Mahmoudian, L.; Gibbs, T.; Ivanov, I.; Malvea, A.; Stojcic, J.; Allan, A. L.; Lowes, L. E.; Sargent, E. H.; Nam, R. K.; Kelley, S. O. Nanoparticle-Mediated Binning and Profiling of Heterogeneous Circulating Tumor Cell Subpopulations. Angew. Chem. 2015, 127 (1), 141-145.

(3) Poudineh, M.; Aldridge, P. M.; Ahmed, S.; Green, B. J.; Kermanshah, L.; Nguyen, V.; Tu, C.; Mohamadi, R. M.; Nam, R. K.; Hansen, A.; Sridhar, S. S.; Finelli, A.; Fleshner, N. E.; Joshua, A. M.; Sargent, E. H.; Kelley, S. O. Tracking the Dynamics of Circulating Tumour Cell Phenotypes using Nanoparticle-mediated Magnetic Ranking. Nat. Nanotechnol. 2016, 12 (3), 274-281.

(4) Wang, L.; Yang, Z.; Gao, J.; Xu, K.; Gu, H.; Zhang, B.; Zhang, X.; $\mathrm{Xu}, \mathrm{B}$. A Biocompatible Method of Decorporation: BisphosphonateModified Magnetite Nanoparticles to Remove Uranyl Ions from Blood. J. Am. Chem. Soc. 2006, 128 (41), 13358-13359.

(5) Lee, H. Y.; Bae, D. R.; Park, J. C.; Song, H.; Han, W. S.; Jung, J. H. A Selective Fluoroionophore Based on BODIPY-functionalized Magnetic Silica Nanoparticles: Removal of $\mathrm{Pb} 2+$ from Human Blood. Angew. Chem., Int. Ed. 2009, 48 (7), 1239-1243.

(6) Herrmann, I. K.; Urner, M.; Koehler, F. M.; Hasler, M.; RothZ'Graggen, B.; Grass, R. N.; Ziegler, U.; Beck-Schimmer, B.; Stark, W. J. Blood Purification using Functionalized Core/Shell Nanomagnets. Small 2010, 6 (13), 1388-1392.

(7) Cai, K.; Li, J.; Luo, Z.; Hu, Y.; Hou, Y.; Ding, X. [small beta]Cyclodextrin conjugated Magnetic Nanoparticles for Diazepam Removal from Blood. Chem. Commun. 2011, 47 (27), 7719-7721.

(8) Shen, H.; Wang, J.; Liu, H.; Li, Z.; Jiang, F.; Wang, F.-B.; Yuan, Q. Rapid and Selective Detection of Pathogenic Bacteria in Bloodstream Infections with Aptamer-Based Recognition. ACS Appl. Mater. Interfaces 2016, 8 (30), 19371-19378.

(9) Kang, J. H.; Super, M.; Yung, C. W.; Cooper, R. M.; Domansky, K.; Graveline, A. R.; Mammoto, T.; Berthet, J. B.; Tobin, H.; Cartwright, M. J.; Watters, A. L.; Rottman, M.; Waterhouse, A.; Mammoto, A.; Gamini, N.; Rodas, M. J.; Kole, A.; Jiang, A.; Valentin, T. M.; Diaz, A.; Takahashi, K.; Ingber, D. E. An extracorporeal Bloodcleansing Device for Sepsis Therapy. Nat. Med. 2014, 20 (10), 12111216.

(10) Lattuada, M.; Ren, Q.; Zuber, F.; Galli, M.; Bohmer, N.; Matter, M. T.; Wichser, A.; Bertazzo, S.; Pier, G. B.; Herrmann, I. K. Theranostic Body Fluid Cleansing: rationally designed Magnetic Particles enable Capturing and Detection of Bacterial Pathogens. J. Mater. Chem. B 2016, 4 (44), 7080-7086.

(11) Molday, R. S.; Yen, S. P. S.; Rembaum, A. Application of Magnetic Microspheres in Labelling and Separation of Cells. Nature 1977, 268 (5619), 437-438.

(12) Miltenyi, S.; Müller, W.; Weichel, W.; Radbruch, A. High gradient Magnetic Cell Separation with MACS. Cytometry 1990, 11 (2), 231-238.

(13) Molday, R. S.; Mackenzie, D. Immunospecific Ferromagnetic Iron-dextran Reagents for the Labeling and Magnetic Separation of Cells. J. Immunol. Methods 1982, 52 (3), 353-367.

(14) Xu, H.; Aguilar, Z. P.; Yang, L.; Kuang, M.; Duan, H.; Xiong, Y.; Wei, H.; Wang, A. Antibody conjugated Magnetic Iron Oxide Nanoparticles for Cancer Cell Separation in fresh Whole Blood. Biomaterials 2011, 32 (36), 9758-9765.

(15) Green, B. J.; Saberi Safaei, T.; Mepham, A.; Labib, M.; Mohamadi, R. M.; Kelley, S. O. Beyond the Capture of Circulating Tumor Cells: Next-Generation Devices and Materials. Angew. Chem., Int. Ed. 2016, 55 (4), 1252-1265. 
(16) Khoo, B. L.; Warkiani, M. E.; Tan, D. S.-W.; Bhagat, A. A. S.; Irwin, D.; Lau, D. P.; Lim, A. S. T.; Lim, K. H.; Krisna, S. S.; Lim, W.T.; Yap, Y. S.; Lee, S. C.; Soo, R. A.; Han, J.; Lim, C. T. Clinical Validation of an Ultra High-Throughput Spiral Microfluidics for the Detection and Enrichment of Viable Circulating Tumor Cells. PLoS One 2014, 9 (7), e99409.

(17) Nagrath, S.; Sequist, L. V.; Maheswaran, S.; Bell, D. W.; Irimia, D.; Ulkus, L.; Smith, M. R.; Kwak, E. L.; Digumarthy, S.; Muzikansky, A.; Ryan, P.; Balis, U. J.; Tompkins, R. G.; Haber, D. A.; Toner, M. Isolation of Rare Circulating Tumour Cells in Cancer Patients by Microchip Technology. Nature 2007, 450 (7173), 1235-1239.

(18) Stott, S. L.; Hsu, C.-H.; Tsukrov, D. I.; Yu, M.; Miyamoto, D. T.; Waltman, B. A.; Rothenberg, S. M.; Shah, A. M.; Smas, M. E.; Korir, G. K.; Floyd, F. P.; Gilman, A. J.; Lord, J. B.; Winokur, D.; Springer, S.; Irimia, D.; Nagrath, S.; Sequist, L. V.; Lee, R. J.; Isselbacher, K. J.; Maheswaran, S.; Haber, D. A.; Toner, M. Isolation of Circulating Tumor Cells using a Microvortex-generating Herringbonechip. Proc. Natl. Acad. Sci. U. S. A. 2010, 107 (43), 18392-18397.

(19) Yamamoto, S.; Shimizu, K.; Fei, J.; Iwata, H.; Okochi, M.; Nakanishi, H.; Honda, H. Ex vivo Culture of Circulating Tumor Cells using Magnetic Force-based Coculture on a Fibroblast Feeder Layer. Biotechnol. J. 2016, 11 (11), 1433-1442.

(20) Yoon, Y.; Cho, S.; Kim, S.; Choi, E.; Kim, R. K.; Lee, S. J.; Sul, O.; Lee, S. B. Separation and Capture of Circulating Tumor Cells from Whole Blood using a Bypass integrated Microfluidic Trap Array. In 36th Annual International Conference of the IEEE Engineering in Medicine and Biology Society, 26-30 Aug. 2014; 2014; pp 4431-4434.

(21) Scarberry, K. E.; Mezencev, R.; McDonald, J. F. Targeted Removal of Migratory Tumor Cells by Functionalized Magnetic Nanoparticles impedes Metastasis and Tumor Progression. Nanomedicine 2011, 6 (1), 69-78.

(22) Herrmann, I. K.; Bernabei, R. E.; Urner, M.; Grass, R. N.; BeckSchimmer, B.; Stark, W. J. Device for Continuous Extracorporeal Blood Purification using Target-specific Metal Nanomagnets. Nephrol., Dial., Transplant. 2011, 26 (9), 2948-U1516.

(23) Allard, W. J.; Matera, J.; Miller, M. C.; Repollet, M.; Connelly, M. C.; Rao, C.; Tibbe, A. G. J.; Uhr, J. W.; Terstappen, L. W. M. M. Tumor Cells Circulate in the Peripheral Blood of All Major Carcinomas but not in Healthy Subjects or Patients With Nonmalignant Diseases. Clin. Cancer Res. 2004, 10 (20), 6897-6904.

(24) Kang, J. H.; Um, E.; Diaz, A.; Driscoll, H.; Rodas, M. J.; Domansky, K.; Watters, A. L.; Super, M.; Stone, H. A.; Ingber, D. E. Optimization of Pathogen Capture in Flowing Fluids with Magnetic Nanoparticles. Small 2015, 11 (42), 5657-5666.

(25) Herrmann, I. K.; Schlegel, A.; Graf, R.; Schumacher, C. M.; Senn, N.; Hasler, M.; Gschwind, S.; Hirt, A.-M.; Guenther, D.; Clavien, P.-A.; Stark, W. J.; Beck-Schimmer, B. Nanomagnet-based Removal of Lead and Digoxin from Living Rats. Nanoscale 2013, 5 (18), 8718-8723.

(26) Yu, M.; Stott, S.; Toner, M.; Maheswaran, S.; Haber, D. A. Circulating Tumor Cells: Approaches to Isolation and Characterization. J. Cell Biol. 2011, 192 (3), 373-382.

(27) Rossier, M.; Koehler, F. M.; Athanassiou, E. K.; Grass, R. N.; Waelle, M.; Birbaum, K.; Günther, D.; Stark, W. J. Energy-Efficient Noble Metal Recovery by the Use of Acid-Stable Nanomagnets. Ind. Eng. Chem. Res. 2010, 49 (19), 9355-9362. 